\title{
On Convolution and Convex Combination of Harmonic Mappings
}

\author{
Ahmad Sulaiman Ahmad El-Faqeer $\mathbb{D}$, Zhen Chuan Ng $\mathbb{D}$, and Shamani Supramaniam
}

School of Mathematical Sciences, Universiti Sains Malaysia, Penang 11800, Malaysia

Correspondence should be addressed to Zhen Chuan Ng; zhenchuanng@usm.my

Received 17 May 2021; Revised 7 July 2021; Accepted 23 July 2021; Published 12 August 2021

Academic Editor: V. Ravichandran

Copyright (C) 2021 Ahmad Sulaiman Ahmad El-Faqeer et al. This is an open access article distributed under the Creative Commons Attribution License, which permits unrestricted use, distribution, and reproduction in any medium, provided the original work is properly cited.

In this paper, the subclass of harmonic univalent functions by shearing construction is studied and this subclass of harmonic mappings needs a necessary and adequate condition to be convex in the horizontal direction. Furthermore, convolutions of two special subclasses of univalent harmonic mappings are shown to be convex in the horizontal direction. Also, the family of univalent harmonic mappings of the unit disk onto a region convex in the direction of the imaginary axis is introduced. Sufficient conditions for convex combinations of harmonic mappings of this family to be univalently convex in the direction of the imaginary axis are obtained.

\section{Motivation and Preliminaries}

A complex-valued function $f=u+i v$ defined on the unit $\operatorname{disk} U=\{z \in \mathbb{C}:|z|<1\}$ is called harmonic mapping if $u$ and $v$ are real-valued harmonic functions. In addition, since $U$ is a simply connected domain, $f$ has a unique representation $f=h+\bar{g}$, where $h$ and $g$ are analytic and co-analytic parts of $f$, respectively. It is known from Lewy [1] that the mapping $f=h+\bar{g}$ defined on $U$ is locally univalent and sense-preserving if and only if

$$
\left|g^{\prime}(z)\right|<\left|h^{\prime}(z)\right|, \quad \forall z \in U .
$$

The class of all univalent, harmonic sense-preserving mappings $f=h+\bar{g}$ defined in $U$ is denoted by $\mathscr{H}$. Moreover, let $S_{H}$ be the class of all functions $f \in \mathscr{H}$ which is normalized by $f(0)=f_{z}(0)-1=0$. Therefore, each member of $f \in S_{H}$ has the following representation:

$$
f(z)=z+\sum_{n=2}^{\infty} b_{n} z^{n}+\sum_{n=1}^{\infty} \bar{c}_{n} \bar{z}^{n}\left(\left|c_{1}\right|<1\right),
$$

for all $z \in U$.

The class of such type of functions given in (2) with $c_{1}=$ $0=f_{\bar{z}}(0)$ is a subclass of $S_{H}$ and is denoted by $S_{H}^{0}$. The dilatation of $f$ belonging to $S_{H}^{0}$ is the function $\omega: U \longrightarrow \mathbb{C}$ given by $\omega=g^{\prime}(z) / h^{\prime}(z)$ and satisfies $|\omega(z)|<1$ for all $z \in U$. Further, denote by $K_{H}$ (or $K_{H}^{0}$ ) all $f \in S_{H}$ (or $S_{H}^{0}$ ) which are mapping $U$ onto convex regions. For comprehensive and fundamental knowledge on planar harmonic mappings, see Duren [2]. The subclass of $S_{H}^{0}$ denoted by $S_{\mathrm{CHD}}^{0}$ consists of all univalent harmonic functions which maps onto domain convex in the direction of the real axis.

Section 2 demonstrates that the convolution of two special subclasses of univalent harmonic mappings is convex in the horizontal direction. The convolution of any two arbitrary harmonic functions

$$
\begin{aligned}
& f(z)=h(z)+\bar{g}(z)=z+\sum_{n=2}^{\infty} a_{n} z^{n}+\sum_{n=1}^{\infty} \bar{b}_{n} \bar{z}^{n}, \\
& F(z)=H(z)+\bar{G}(z)=z+\sum_{n=2}^{\infty} A_{n} z^{n}+\sum_{n=1}^{\infty} \bar{B}_{n} \bar{z}^{n}
\end{aligned}
$$

is defined by

$$
\begin{aligned}
(f \widetilde{*})(z)= & (h * H)(z)+\overline{(g * G)}(z)=z+\sum_{n=2}^{\infty} a_{n} A_{n} z^{n} \\
& +\sum_{n=1}^{\infty} \bar{b}_{n} \bar{B}_{n} \bar{z}^{n} .
\end{aligned}
$$


The convolution of harmonic functions is different from the convolution of univalent functions (for more details, one can refer to [3]). Indeed, the convolution of two harmonic functions does not preserve the convexity and the convolution of any two univalent harmonic functions $f \in S_{H}^{0}$ need not be univalent. These facts generated significant interest in the analysis of harmonic convolutions of univalent harmonic functions, and several articles on this subject recently appeared in the literature [3-11]. Specifically, the collection of the mappings $F=H+\bar{G} \in S_{H}^{0}$ that map $U$ onto the right half-plane $\Psi=\{w: \operatorname{Re}(w)>-(1 / 2)\}$ and have the form $(H+G)(z)=z /(1-z)$ with $\left|G^{\prime}\right|<\left|H^{\prime}\right|$ for all $z \in U$. Kumar et al. [12] proposed a class of right half-plane harmonic mappings, $F_{a}=H_{a}+\bar{G}_{a},(-1<a<1)$, satisfying

$$
\begin{aligned}
& H_{a}(z)+G_{a}(z)=\frac{z}{1-z}, \\
& G_{a}^{\prime}(z)=\frac{a-z}{1-a z} H_{a}^{\prime}(z) .
\end{aligned}
$$

Using the shear construction of Clunie and Sheil-Small [13], it follows that

$$
\begin{aligned}
& H_{a}(z)=\frac{z /(1+a)-1 / 2 z^{2}}{(1-z)^{2}}, \\
& G_{a}(z)=\frac{(a / 1+a) z-(1 / 2) z^{2}}{(1-z)^{2}} .
\end{aligned}
$$

Letting $a=0$ in (5), the mapping $F_{0}=H_{0}+G_{0}$ with $\left(F_{0}+G_{0}\right)(z)=z /(1-z)$ and $G_{0}^{\prime}(z)=-z H_{0}^{\prime}(z)$, which is called the standard right half-plane mapping, is obtained. In [9], the following result was obtained.

Theorem 1 (see [9]). Let $f=h+\bar{g} \in K_{H}^{0}$ satisfy the condition $(h+g)(z)=z /(1-z)$ and dilatation $\omega(z)=g^{\prime}(z) /$ $h^{\prime}(z)=e^{i \theta} z^{n}\left(n \in \mathbb{Z}^{+} ; \theta \in \mathbb{R}\right)$. If $n=1,2$, then $F_{0} * f \in S_{C H D}^{0}$.

Recently, Liu and Ponnusamy [8] generalized Theorem 1 in the case $n=1$ as follows.

Theorem 2 (see [8]). Let $f=h+\bar{g} \in K_{H}^{0}$ satisfy the condition $(h+g)(z)=(1+a) z /(1-z)$ with dilatation $\omega_{1}(z)=(z+a) /(1+a z)$, where $a \in(-1,1)$, and $f_{1}=h_{1}+$ $\bar{g}_{1} \in K_{H}^{0}$ with dilatation $\omega_{2}(z)=e^{i \theta} z(\theta \in \mathbb{R})$. Then, $f *$ $f_{1} \in S_{C H D}^{0}$.

Liu and Ponnusamy [8] also proposed the following problem.

Problem 1. Let $f=h+\bar{g} \in K_{H}$ with $(h+g)(z)=$ $(1+a) z /(1-z)$ and dilatation function $\omega_{1}(z)=(z+a) /$ $(1+a z)$, where $a \in(-1,1)$, and let $f_{n}=h_{n}+\bar{g}_{n} \in K_{H}^{0}$ with dilatation $\omega_{2}(z)=e^{i \theta} z^{n}\left(\theta \in \mathbb{R}, n \in \mathbb{N}^{+}\right)$. Then, $f * f_{n} \in$ $S_{\mathrm{CHD}}^{0}$.

Ali et al. [14] solved Problem 1 for $\omega_{2}(z)=-z$. In [8], the authors conjectured that $f * f_{n}$ is not locally univalent for $n \geq 2$. In Section 2, new subclass of harmonic mappings $P_{a}[\phi]$ is obtained. It is also shown that the necessary and sufficient condition for $P_{a}[\phi] \in S_{\mathrm{CHD}}^{0}$, if and only if $\phi(z)$ is convex. The function $f_{n}=h+\bar{g}$ satisfies the condition $(h-$ $g)(z)=z /(1-z)$ and dilatation $\omega(z)=z^{n} ; n \in \mathbb{N}$ with the mapping $P_{a}[\ell]$ belonging to subclass $S_{\mathrm{CHD}}^{0}$ for $a \in(-1,(2-$ $n) /(2+n))$ and for all $n \in \mathbb{N}$ are also explored.

The results proposed by Hengartner and Schober [15] and Pommenrenke [16] are very useful in checking the convexity of an analytic function in the direction of the imaginary axis as well as the convexity in the direction of the real axis, respectively.

Lemma 1 (see [15]). Suppose $f$ is a nonconstant analytic function of $U$. Then,

$$
\operatorname{Re}\left\{\left(1-z^{2}\right) f^{\prime}(z)\right\} \geq 0
$$

if and only if

(1) $f$ is univalent in $U$.

(2) $f$ is convex in the direction of imaginary axis.

(3) There exist sequences $\left\{z_{n}^{\prime}\right\}$ converging to $z=1$ and $\left\{z_{n}^{\prime \prime}\right\}$ converging to $z=-1$, such that

$$
\begin{aligned}
& \lim _{n \longrightarrow \infty} \operatorname{Re}\left\{f\left(z_{n}^{\prime}\right)\right\}=\sup _{|z|<1} \operatorname{Re}(f(z)), \\
& \lim _{n \longrightarrow \infty} \operatorname{Re}\left\{f\left(z_{n}^{\prime \prime}\right)\right\}=\inf _{|z|<1} \operatorname{Re}(f(z)) .
\end{aligned}
$$

Lemma 2 (see [16]). Suppose $f$ is a nonconstant analytic function of $U$ satisfying the condition $f(0)=0$ and $f^{\prime}(0)=0$, and suppose

$$
\varphi(z)=\frac{z}{\left(1+z e^{i \theta_{1}}\right)\left(1+z e^{i \theta_{2}}\right)},
$$

where $\theta_{i}, i=1,2 \in \mathbb{R}$. If

$$
\operatorname{Re}\left(\frac{z f^{\prime}(z)}{\varphi(z)}\right)>0,
$$

then $f$ is convex in the direction of the real axis.

Recall that the region $\Omega$ is said to be convex in the direction $\theta, 0 \leq \theta<\pi$, if every line parallel to the line through 0 and $e^{i \theta}$ has either connected or empty intersection with $\Omega$, and if $\Omega$ is convex in every direction, then it is called convex. If $\theta=0(\operatorname{or} \theta=\pi / 2)$, then $\Omega$ is convex in the direction of the real axis "CHD" or (is convex in the direction of the imaginary axis "CID"). Clunie and Sheil [13] proposed an integrated way to construct a univalent harmonic mapping convex in a given direction.

Theorem 3 (see [13]). Let $0 \leq \theta<\pi$. A locally univalent harmonic function $f=h+\bar{g}$ in $U$ is a univalent mapping of $U$ onto a domain convex in the direction of $\theta$ if and only if $h-e^{2 i \theta} g$ is a univalent mapping of $U$ onto a domain convex in the direction of $\theta$.

The study of the geometric properties of the convex combination of the univalent mappings is another important topic in the geometric function theory. Dorff and Rolf [17] developed a necessary condition for convex combination to be 
univalent maps onto a domain convex in the direction of the imaginary axis.

Theorem 4 (see [17]). Let $f_{1}=h_{1}+\bar{g}_{1}$ and $f_{2}=h_{2}+\bar{g}_{2}$ be two univalent harmonic mappings defined on $U$, with $f_{1}$ and $f_{2}$ having the same second complex dilatation and satisfying conditions in (4); then,

$$
\begin{aligned}
f_{3} & =h_{3}+\bar{g}_{3} \\
& =\left[t h_{1}(z)+(1-t) h_{2}\right]+\left[t \bar{g}_{1}(z)+(1-t) \bar{g}_{2}(z)\right] \\
& =t f_{1}(z)+(1-t) f_{2}
\end{aligned}
$$

is univalent and is convex in the direction of the imaginary axis.

It is known that even if $f$ and $g$ are convex analytic functions, the convex combination of $f$ and $g$ may not be a univalent function (see [18], and for more recent studies of linear combinations of harmonic mappings, see [17, 19-23]). Moreover, Kumar et al. [20] studied the convexity of linear combination of harmonic mappings, which are shears of the analytic mapping $z /(1-z)$ and $z(1-\beta z) /\left(1-z^{2}\right)$, for $-1 \leq \beta \leq 1$. Beig et al. [23] studied and found necessary conditions for the convex combination of the right half-plane mappings, the vertical strip mapping, their rotations, and some other harmonic mappings to be univalent and convex in a particular direction. In Section 3, the convex combination of mappings of the family of sense-preserving and locally univalent harmonic mappings $f_{\alpha}=h_{\alpha}+g_{\alpha}$, by shearing the function $h_{\alpha}+g_{\alpha}=K_{\alpha}$ where

$$
\begin{aligned}
K_{\alpha}(z) & =h_{\alpha}(z)+g_{\alpha}(z)=\frac{z\left(1-\alpha z^{2}\right)}{1-z^{2}}, \quad \alpha \in[0,1], \\
\left|\frac{g_{\alpha}^{\prime}}{h_{\alpha}^{\prime}}\right| & <1,
\end{aligned}
$$

are studied. Further, the appropriate conditions for the convex combination in the vertical direction between univalent harmonic mappings to be univalent and convex in the vertical direction are also established.

\section{Convolutions of Subclasses of Univalent Harmonic Right Half-Plane Mappings}

In this section, we consider the harmonic mapping $P_{a}=$ $h_{a}+\bar{g}_{a}$ with $\left(h_{a}-g_{a}\right)(z)=(1-a) z /(1-z)$ with the second complex dilatation $\omega(z)=(a+z) /(1+a z)$, $a \in(-1,1)$. Hence, we can solve for $h_{a}$ and $g_{a}$ as follows:

$$
\begin{aligned}
& h_{a}(z)=\left(\frac{z-(1 / 2)(1-a) z^{2}}{(1-z)^{2}}\right)=\frac{1}{2}\left(\frac{(1+a) z}{(1-z)^{2}}+\frac{(1-a) z}{(1-z)}\right), \\
& g_{a}(z)=h_{a}(z)-\frac{(1-a) z}{1-z}=\frac{1}{2}\left(\frac{(1+a) z}{(1-z)^{2}}-\frac{(1-a) z}{(1-z)}\right),
\end{aligned}
$$

and thus

$$
\begin{aligned}
P_{a}(z)= & h_{a}(z)+\bar{g}_{a}(z)=\frac{1}{2}\left(\left(\frac{(1+a) z}{(1-z)^{2}}+\frac{(1-a) z}{(1-z)}\right)\right. \\
& +\left(\overline{(1+a) z} \frac{(1-a) z}{(1-z)^{2}}-\frac{(1-z)}{(1-z)} .\right.
\end{aligned}
$$

Taking $\ell(z)=z /(1-z), P_{a}$ given in (14) can be rewritten as

$$
\begin{aligned}
P_{a}(z)= & \frac{1}{2}\left(\left((1+a) z \ell^{\prime}(z)+(1-a) \ell(z)\right)\right. \\
& \left.+\left(\overline{(1+a) z \ell^{\prime}(z)-(1-a) \ell(z)}\right)\right) .
\end{aligned}
$$

For an analytic univalent function $\phi: U \longrightarrow \mathbb{C}$ normalized by $\phi(0)=\phi^{\prime}(0)-1=0$, define

$$
\begin{aligned}
P_{a}[\phi](z)= & \frac{1}{2}\left(\left((1+a) z \phi^{\prime}(z)+(1-a) \phi(z)\right)\right. \\
& \left.+\left(\overline{(1+a) z \phi^{\prime}(z)-(1-a) \phi(z)}\right)\right) \\
= & H_{a}[\phi](z)+\bar{G}_{a}[\phi](z),
\end{aligned}
$$

for all $z \in U$. It is clear that $P_{a}[\ell]=P_{a}$. Therefore,

$$
\begin{aligned}
P_{a}[\ell](z)= & \frac{1}{2}\left(\left((1+a) z \ell^{\prime}(z)+(1-a) \ell(z)\right)\right. \\
& \left.+\left(\overline{(1+a) z \ell^{\prime}(z)-(1-a) \ell(z)}\right)\right) \\
= & H_{a}(z)+\bar{G}_{a}(z) .
\end{aligned}
$$

If $F$ is an analytic function with $F(0)=0$, then

$$
\left(H_{a} * F\right)(z)=\frac{1}{2}\left((1+a) z F^{\prime}(z)+(1-a) F(z)\right),
$$

and

$$
\left(G_{a} * F\right)(z)=\frac{1}{2}\left((1+a) z F^{\prime}(z)-(1-a) F(z)\right) .
$$

Lemma 3. Let $f=h+\bar{g}$ satisfy the condition $(h-g)(z)=$ $z /(1-z)$ with second complex dilatation $\omega=g^{\prime} / h^{\prime}$ such that $|\omega|<1$ and $P_{a}[\ell]$ be family of harmonic mappings given in (17). Then, $\widehat{\omega}$, the second complex dilatation of $f * P_{a}[\ell]$, is given by

$$
\widehat{\omega}(z)=\frac{2(a+z) \omega(1-\omega)+(1+a) z \omega^{\prime}(1-z)}{2(1+a z)(1-\omega)+(1+a) z \omega \prime(1-z)} .
$$

Proof. Since

$$
f * P_{a}[\ell]=(h+\bar{g}) *\left(H_{a}+\bar{G}_{a}\right)=\left(h * H_{a}\right)+\overline{\left(g * G_{a}\right)},
$$

then 


$$
\widehat{\omega}(z)=\frac{\left(g * G_{a}\right)^{\prime}(z)}{\left(h * H_{a}\right)^{\prime}(z)} .
$$

Now, from (18) and (19), it follows that

$$
\begin{aligned}
\widehat{\omega}(z) & =\frac{\left((1+a) z g^{\prime}(z)-(1-a) g(z)\right)^{\prime}}{\left((1+a) z h^{\prime}(z)+(1-a) h(z)\right)^{\prime}} \\
& =\frac{2 a g^{\prime}(z)+(1+a) z g^{\prime \prime}(z)}{2 h^{\prime}(z)+(1+a) z h^{\prime \prime}} .
\end{aligned}
$$

Since $g^{\prime \prime}=\omega^{\prime} h^{\prime}+\omega h^{\prime \prime}$, then

$$
\widehat{\omega}(z)=\frac{\left(2 a \omega+(1+a) z \omega^{\prime}\right) h^{\prime}(z)+(1+a) z \omega h^{\prime \prime}(z)}{2 h^{\prime}(z)+(1+a) z h^{\prime \prime}} .
$$

From $(h-g)(z)=z /(1-z)$, it follows that

$$
\begin{aligned}
h^{\prime}(z) & =\frac{1}{(1-\omega)(1-z)^{2}}, \\
h^{\prime \prime}(z) & =\frac{2(1-\omega)+\omega^{\prime}(1-z)}{(1-\omega)^{2}(1-z)^{3}},
\end{aligned}
$$

and thus

$$
\begin{aligned}
\widehat{\omega}(z) & =\frac{\left(2 a \omega+(1+a) z \omega^{\prime}\right)(1-\omega)(1-z)+(1+a) z \omega\left[2(1-\omega)+\omega^{\prime}(1-z)\right]}{2(1-\omega)(1-z)+(1+a) z\left[2(1-\omega)+\omega^{\prime}(1-z)\right]} \\
& =\frac{2(a+z) \omega(1-\omega)+(1+a) z \omega^{\prime}(1-z)}{(2+2 a z)(1-\omega)+(1+a) z \omega^{\prime}(1-z)} .
\end{aligned}
$$

Lemma 4. Let $f=h+\bar{g} \in S_{H}^{0}$ with $(h-g)(z)=z /(1-z)$. If $P_{a}[\ell] * f$ is locally univalent, then $P_{a}[\ell] * f$ is in $S_{C H D}^{0}$.

Proof. Since

$$
\begin{aligned}
H_{a}-G_{a} & =(1+a) \ell(z), \\
h-g & =\ell(z),
\end{aligned}
$$

then

$$
\begin{aligned}
(1+a)(h+g) & =\left(H_{a}-G_{a}\right) *(h+g) \\
& =\left(H_{a} * h+H_{a} * g-G_{a} * h-G_{a} * g\right), \\
\left(H_{a}+G_{a}\right) & =\left(H_{a}+G_{a}\right) *(h-g) \\
& =\left(H_{a} * h\right)-\left(H_{a} * g\right)+\left(G_{a} * h\right)-\left(G_{a} * g\right) .
\end{aligned}
$$

Thus,

$$
\left(H_{a} * h\right)-\left(G_{a} * g\right)=\frac{1}{2}\left[(1+a)(h+g)+\left(H_{a}+G_{a}\right)\right],
$$

$$
\left(\left(H_{a} * h\right)-\left(G_{a} * g\right)\right)^{\prime}=\frac{1}{2}\left[(1+a)\left(h^{\prime}+g^{\prime}\right)+\left(H_{a}^{\prime}+G_{a}^{\prime}\right)\right] .
$$

Let $\varphi(z)=z /(1-z)^{2}$. Consequently, we have

$$
\begin{aligned}
& \operatorname{Re}\left(\frac{z\left(H_{a} * h-G_{a} * g\right)^{\prime}}{\varphi}\right) \\
= & \operatorname{Re}\left(\frac{(1+a) z}{2 \varphi}\left(h^{\prime}+g^{\prime}\right)+\frac{z}{2 \varphi}\left(H_{a}^{\prime}+G_{a}^{\prime}\right)\right) \\
= & \frac{1}{2}\left[\operatorname{Re}\left((1+a) \frac{z}{\varphi}\left(h^{\prime}-g^{\prime}\right) \frac{\left(h^{\prime}+g^{\prime}\right)}{\left(h^{\prime}-g^{\prime}\right)}\right)+\operatorname{Re}\left(\frac{z}{\varphi}\left(H_{a}^{\prime}-G_{a}^{\prime}\right) \frac{\left(H_{a}^{\prime}+G_{a}^{\prime}\right)}{\left(H_{a}^{\prime}-G_{a}^{\prime}\right)}\right)\right] \\
= & \frac{(1+a)}{2}\left[\operatorname{Re}\left(\frac{h^{\prime}+g^{\prime}}{h^{\prime}-g^{\prime}}\right)+\operatorname{Re}\left(\frac{H_{a}^{\prime}+G_{a}^{\prime}}{H_{a}^{\prime}-G_{a}^{\prime}}\right)\right] \\
= & \frac{(1+a)}{2}\left[\operatorname{Re} p_{1}(z)+\operatorname{Re} p_{2}(z)\right]>0,
\end{aligned}
$$


where $p_{1}(z)=\left(1+\omega_{f}\right) /\left(1-\omega_{f}\right)$ and $p_{2}(z)=\left(1+\omega_{P_{a}[\ell]}\right) /$ $\left(1-\omega_{P_{a}[\ell]}\right)$. Thus, from Lemma 3 , we deduce that $\left(H_{a} * h\right)-$ $\left(G_{a} * g\right)$ is convex in the direction of the real axis. Theorem 3 implies that $P_{a}[\ell] * f \in S_{\mathrm{CHD}}^{0}$.

Lemma 5. The mapping $P_{a}[\phi]$ given in (16) is locally univalent if and only if the function $\phi$ is convex.

Proof. The mapping $P_{a}[\phi]=H_{a}[\phi]+\overline{G_{a}}[\phi]$ is given in (16). Since $P_{a}[\phi]$ is locally univalent if and only if $\left|G_{a}^{\prime}[\phi] / H_{a}^{\prime}[\phi]\right|<1$,

$$
\left|\frac{2 a \phi^{\prime}(z)+(1+a) z \phi^{\prime \prime}(z)}{2 \phi^{\prime}(z)+(1+a) z \phi^{\prime \prime}(z)}\right|<1,
$$

and hence

$$
\left|\frac{2 a /(1+a)+z \phi^{\prime \prime}(z) / \phi^{\prime}(z)}{2 /(1+a)+z \phi^{\prime \prime}(z) / \phi^{\prime}(z)}\right|<1
$$

Thus,

$$
\left|\frac{(a-1)}{(1+a)}+\left(1+\frac{z \phi^{\prime \prime}(z)}{\phi^{\prime}(z)}\right)\right|<\left|\frac{(1-a)}{(1+a)}+\left(1+\frac{z \phi^{\prime \prime}(z)}{\phi^{\prime}(z)}\right)\right| \text {. }
$$

It can be easily shown that the above inequality is identical to

$$
\operatorname{Re}\left(1+\frac{z \phi^{\prime \prime}(z)}{\phi^{\prime}(z)}\right)>0
$$

Hence, $P_{a}[\phi]$ is locally univalent if $\phi(z)$ is convex function.

Theorem 5. The mapping $P_{a}[\phi]$ is in the class $S_{C H D}^{0}$, if and only if $\phi$ is convex.

Proof. The mapping $P_{a}[\phi]=H_{a}[\phi]+\bar{G}_{a}[\phi]$ is given in (16), and $H_{a}[\phi]-G_{a}[\phi]=(1-a) \phi$ where $\phi$ is convex. This implies that $H_{a}[\phi]-G_{a}[\phi]$ is convex in the direction of the real axis. Consequently, from Lemma 5 and Theorem 3, we deduce that $P_{a}[\phi]$ in $S_{\mathrm{CHD}}^{0}$.

In order to prove the next result, we will employ the distribution theory of the polynomial function roots of the disc unit. Given a polynomial function of degree $m$ :

$$
p(z)=a_{m} z^{m}+a_{m-1} z^{m-1}+\cdots+a_{1} z+a_{0},
$$

with complex coefficients, the parallel algorithm for discovering polynomial zeros for (35) inside $U$ is worth exploring. Let

$$
p^{*}(z)=z^{n} \overline{p\left(\frac{1}{\bar{z}}\right)}=\bar{a}_{m}+\bar{a}_{m-1} z+\cdots+\bar{a}_{1} z^{m-1}+\bar{a}_{0} z^{m} .
$$

The inverse zeros for (35) and (36) can be easily checked with respect to $U$. We need the following lemma to prove the main theorem.
Lemma 6 (Cohn's rule) (see [24]). Let $p$ be a polynomial function as given in (35) of degree $m$ and let $p^{*}$ be as given in (36). The number of zeros of $p$ in or on the unit circle is denoted by $r$ and $s$, respectively. If $\left|a_{0}\right|<\left|a_{m}\right|$, then

$$
p_{1}(z)=\frac{\bar{a}_{m} p_{1}(z)-a_{0} p^{*}(z)}{z}
$$

is a polynomial function of degree $m-1$ with $r_{1}=r-1$ and $s_{1}=s$, where $r_{1}$ and $s_{1}$ are the number of zeros of the polynomial $p_{1}$ in or on the unite circle, respectively.

Theorem 6. Let $P_{a}[\ell]$ be mapping given in (17) and let $f_{n}=$ $h+\bar{g}$ with $(h-g)(z)=z /(1-z)$ and dilatation $\omega_{1}(z)=z^{n}$, $n \in \mathbb{N}$. Then, $P_{a}[\ell] * f_{n} \in S_{C H D}^{0}$ for $a \in(-1,(2-n) /(2+n))$ and for all $n$ belonging to $\mathbb{N}$.

Proof. According Lemma 4, it suffices to show that the mapping $P_{a}[\ell] * f_{n}$ has dilatation $\omega$ such that $|\omega|<1$ for all $z \in U$. Setting $\omega_{1}(z)=z^{n}$ in (20), we obtain

$$
\begin{aligned}
\omega(z) & =-z^{n} \frac{(a(2+n)+n)+(2-n-a n) z-2 a z^{n}-2 z^{1+n}}{-2-2 a z+(2-n-a n) z^{n}+(a(2+n)+n) z^{1+n}} \\
& =-z^{n} \frac{q(z)}{q^{*}(z)}
\end{aligned}
$$

where

$$
\begin{aligned}
q(z)= & (a(2+n)+n)+(2-n-a n) z-2 a z^{n}-2 z^{1+n} \\
= & a_{0}+a_{1} z+a_{n} z^{n}+a_{n+1} z^{n+1}, \\
q^{*}(z)= & z^{n+1} q \overline{\left(\frac{1}{\bar{z}}\right)}=-2-2 a z+(2-n-a n) z^{n} \\
& +(a(2+n)+n) z^{1+n} \\
= & \bar{a}_{n+1}+\bar{a}_{n}+\bar{a}_{1} z^{n}+\bar{a}_{0} z^{n+1} .
\end{aligned}
$$

If $z_{0} \neq 0$, is zero of the polynomial function $q(z)$, then $1 / z_{0}$ will be zero of the polynomial function $q^{*}$. Hence, we can rewrite $\omega$ as

$$
\omega(z)=-z^{n} \frac{\left(z+A_{1}\right)\left(z+A_{2}\right)\left(z+A_{3}\right), \ldots,\left(z+A_{n+1}\right)}{\left(1+\bar{A}_{1} z\right)\left(1+\bar{A}_{2} z\right)\left(1+\bar{A}_{3} z\right), \ldots,\left(1+\bar{A}_{n+1} z\right)} .
$$

In order to show that $|\omega|<1$ in $U$, it suffices to prove that $\left|A_{i}\right|<1$ for $i=1,2,3, \ldots, n+1$, or equivalently, all the zeros $A_{i}$ for $i=1,2,3, \ldots, n+1$ of the polynomial function $q(z)$ lie in or on the unit circle $|z|=1$.

Note that

$$
\left|a_{0}\right|=|a(2+n)+n|<|2|=\left|a_{n+1}\right|, \quad \forall a \in\left(-1, \frac{2-n}{2+n}\right), n \in \mathbb{N},
$$


so Cohn's rule can be applied on $q$. Consider the polynomial function $q_{1}(z)$ given by

$$
\begin{aligned}
q_{1}(z) & =\frac{\bar{a}_{n+1} q(z)-a_{0} q^{*}(z)}{z} \\
& =(1+a)(-2+n+a(2+n))\left(2+n z^{n-1}-(2+n) z^{n}\right) .
\end{aligned}
$$

It is easy to verify that Cohn's rule is applicable to $q_{1}$ also. So, let a polynomial function $q_{2}$ given by

$$
\begin{aligned}
q_{2}(z) & =\frac{-(2+n) q_{1}(z)-2 q^{*}(z)}{z} \\
& =(1+a)(-2+n+a(2+n))\left(4 n+n^{2}\right)(-1+z) z^{n-2} .
\end{aligned}
$$

Then, the zeros of polynomial function $q_{2}$ lie on $\partial U$ or in $U$. We deduce that all zeros of $q_{1}$ lie inside $U$ or on $\partial U$, and this implies that all the zeros of $q$ lie inside $U$ or on $\partial U$.

Corollary 1. Let $P_{a}[\ell]$ be mapping given in (17) and let $f_{2}=$ $h+\bar{g}$ with $(h-g)(z)=z /(1-z)$ and dilatation $\omega_{1}(z)=$ $-z^{n}, n \in \mathbb{N}$. Then, $P_{a}[\ell] * f_{n} \in S_{C H D}^{0}$ for $a \in(-1,(2-n) /$ $(2+n))$.

The following examples illustrate Theorems 5 and 6.

Example 1. In Theorem 6, if $n=2,(h-g)(z)=z /(1-z)$, and $\omega_{f_{2}}(z)=z^{2}$, then

$$
\begin{aligned}
f_{2}(z)= & h(z)+\bar{g}(z) \\
= & \frac{1}{8}\left(\frac{2 z(3-2 z)}{(1-z)^{2}}+\log \left(\frac{1+z}{1-z}\right)\right) \\
& +\frac{1}{8}\left(\frac{2 z(2 z-1)}{(1-z)^{2}}+\log \left(\frac{1+z}{1-z}\right)\right) .
\end{aligned}
$$

It follows from (17) that

$$
\begin{aligned}
P_{a}[\ell](z)= & h_{a}+\overline{g_{a}}=\frac{1}{2}\left(\left(\frac{(1+a) z}{(1-z)^{2}}+\frac{(1-a) z}{(1-z)}\right)\right. \\
& +\left(\overline{(1+a) z} \frac{(1-a) z}{(1-z)^{2}}\right) \frac{(1-z)}{(1-z)},
\end{aligned}
$$

and $P_{a}[\ell](z) * f_{2}(z)=H(z)+\bar{G}(z)$. Further,

$$
\begin{aligned}
H(z) & =\left(h_{a} * h\right)(z) \\
& =\frac{1}{16}\left[\left(\frac{(1+a) z}{(1-z)^{2}}+\frac{(1-a) z}{(1-z)}\right) *\left(\frac{2 z(3-2 z)}{(1-z)^{2}}+\log \left(\frac{1+z}{1-z}\right)\right)\right] \\
& =\frac{1}{16}\left[\frac{8(1+a) z}{(1-z)^{3}(1+z)}+(1-a)\left(\frac{2 z(3-2 z)}{(1-z)^{2}}+\log \left(\frac{1+z}{1-z}\right)\right)\right] \\
G(z) & =\left(g_{a} * g\right)(z) \\
& =\frac{1}{16}\left[\left(\frac{(1+a) z}{(1-z)^{2}}-\frac{(1-a) z}{(1-z)}\right) *\left(\frac{2 z(2 z-1)}{(1-z)^{2}}+\log \left(\frac{1+z}{1-z}\right)\right)\right] \\
& =\frac{1}{16}\left[\frac{8(1+a) z^{3}}{(1-z)^{3}(1+z)}-(1-a)\left(\frac{2 z(2 z-1)}{(1-z)^{2}}+\log \left(\frac{1+z}{1-z}\right)\right)\right]
\end{aligned}
$$

Figures 1-4, respectively, display representations of concentrated circles within $U$ under the harmonic mapping $P_{a}[\ell](z)$ and concentrated circles under the convolution map $P_{a}[\ell](z) * f_{2}(z)$. We take various values of $a=-0.001,-0.5 \in(-1,0)$.

Example 2. Consider an analytic univalent function $\phi(z)=z+\left(z^{2} / 4\right)$, where $\phi(0)=0$ and $\phi^{\prime}(0)=1$. Since $\phi^{\prime}(z)=1+(z / 2)$ and $\phi^{\prime \prime}(z)=1 / 2$,

$$
\operatorname{Re}\left(1+\frac{z \phi^{\prime \prime}(z)}{\phi^{\prime}(z)}\right)=\operatorname{Re}\left(1+\frac{(1 / 2) z}{(1+(1 / 2) z)}\right) \geq 0 .
$$

This implies that the function $\phi(z)$ maps $U$ into convex region. In view of Theorem 1 , the mapping $P_{a}[\phi](z)$ is in $S_{\mathrm{CHD}}^{0}$ for $-1<a<1$. Now

$$
\begin{aligned}
P_{a}[\phi](z)= & \frac{1}{2}\left((1-a)\left(\frac{z^{2}}{4}+z\right)+(a+1)\left(\frac{z^{2}}{2}+z\right)\right) \\
& +\frac{1}{2\left((a+1)\left(\frac{z^{2}}{2}+z\right)-(1-a)\left(\frac{z^{2}}{4}+z\right)\right)}, \quad(z \in U) .
\end{aligned}
$$

Figures 5-7 display images of $U$ under $P_{a}\left[z+(1 / 4) z^{2}\right]$ for various values of $a \in(-1,1)$, respectively. The image of 


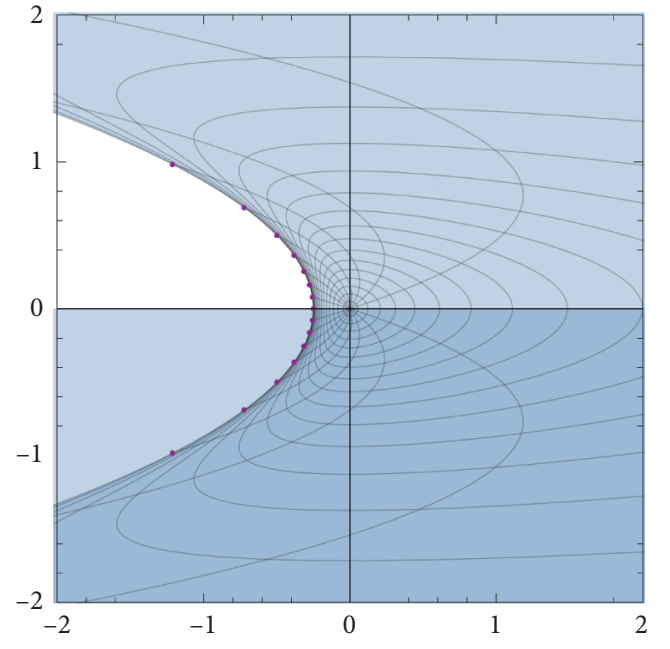

Figure 1: Image of $P_{-0.001}[\ell](z)$.

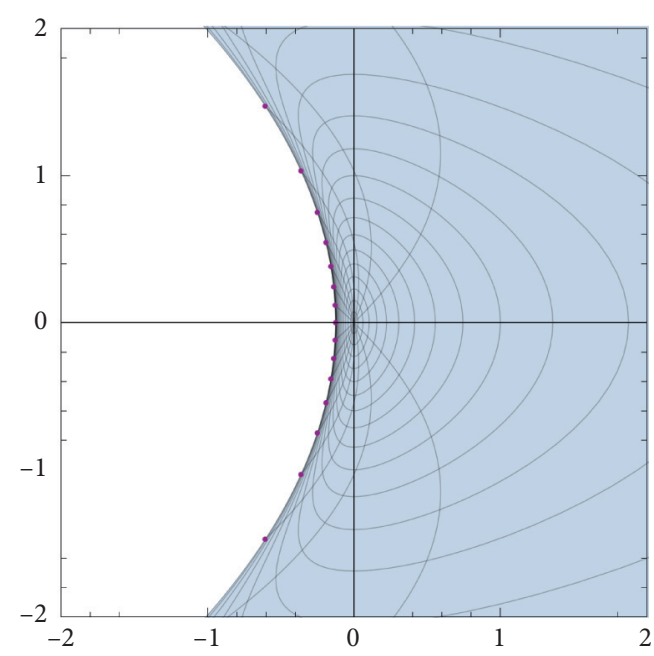

Figure 2: Image of $P_{-0.5}[\ell](z)$.

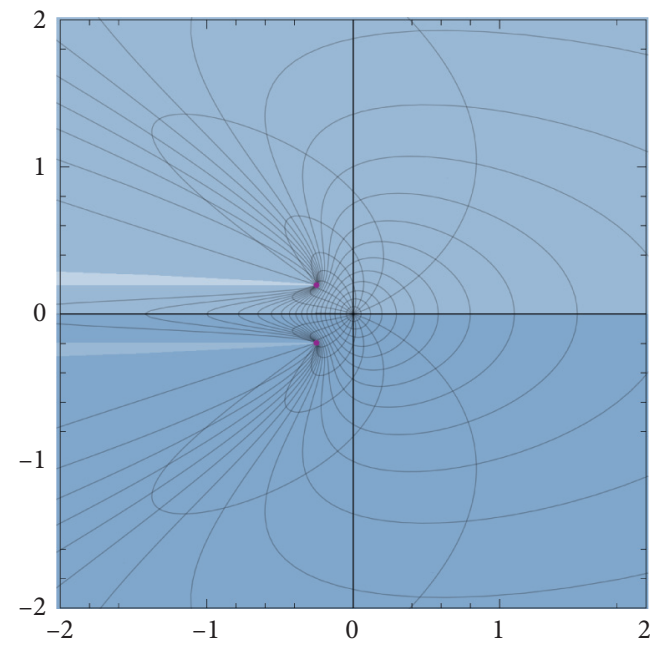

Figure 3: Image of $P_{-0.001}[\ell](z) * f_{2}(z)$.
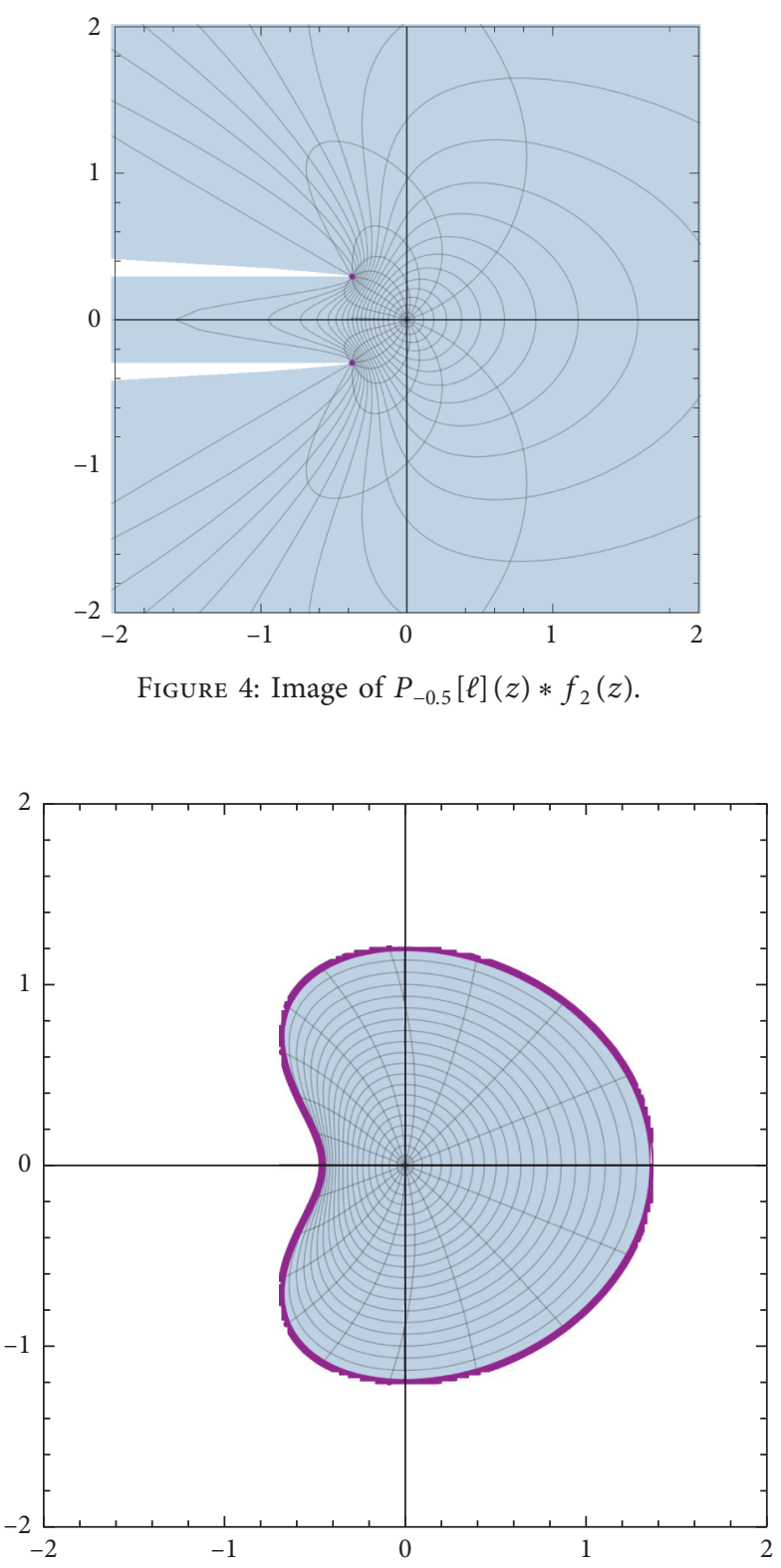

Figure 5: Image of $P_{-0.09}\left[z+\left(z^{2} / 4\right)\right]$.

$U$ under $P_{a}\left[z+(1 / 4) z^{2}\right]$ is convex in the direction of the real axis.

\section{Convex Combination of a Family of Univalent Harmonic Mappings}

Let $f_{\alpha}(z)=h_{\alpha}(z)+\bar{g}_{\alpha}(z)$, where

$$
\begin{aligned}
K_{\alpha}(z) & =h_{\alpha}(z)+g_{\alpha}(z)=\frac{z\left(1-\alpha z^{2}\right)}{1-z^{2}}, \quad \alpha \in[0,1], \\
\left|\frac{g_{\alpha}^{\prime}}{h_{\alpha}^{\prime}}\right| & <1,
\end{aligned}
$$

be normalized, sense-preserving, and locally univalent harmonic mapping defined on $U$. First of all, we have to 


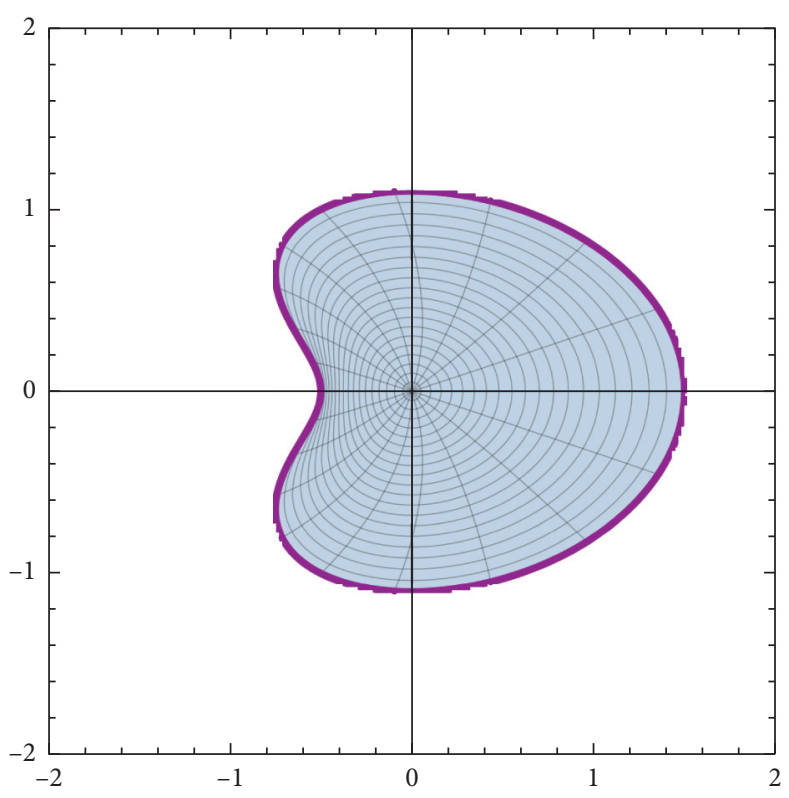

Figure 6: Image of $P_{0}\left[z+\left(z^{2} / 4\right)\right]$.

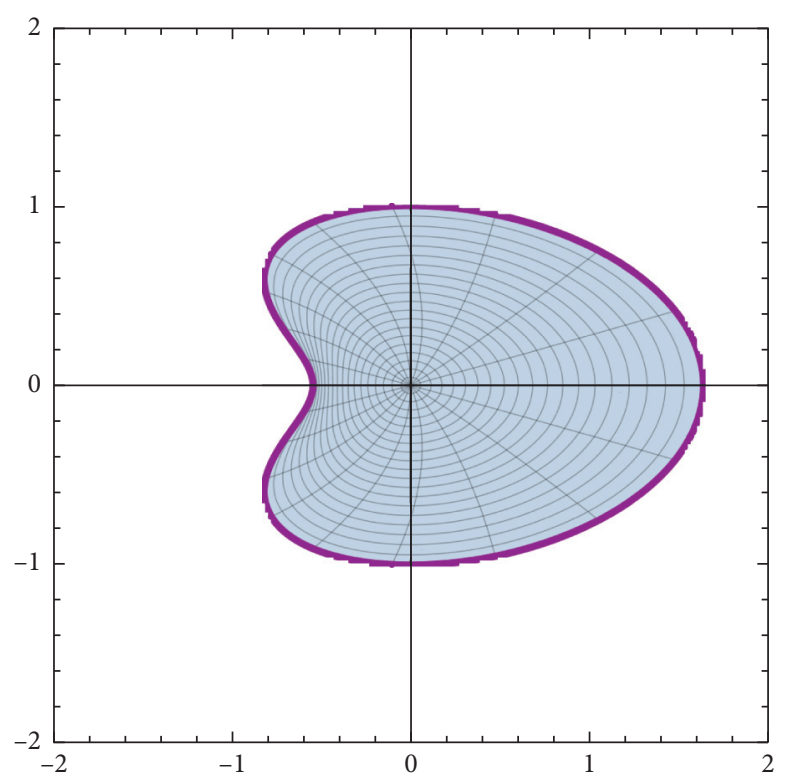

Figure 7: Image of $P_{0.09}\left[z+\left(z^{2} / 4\right)\right]$.

show that $f_{\alpha}$ belongs to class $S_{H}$ and maps onto region convex in the direction of the imaginary axis. Let

$$
\Psi(z)=\left(1-z^{2}\right) K_{\alpha}^{\prime}(z)=\frac{1+\alpha z^{4}+(1-3 \alpha) z^{2}}{1-z^{2}}
$$

for all $z \in U, 0 \leq \alpha \leq 1$, and we will prove that

$$
\operatorname{Re}(\Psi(z))=\operatorname{Re} \frac{1+\alpha z^{4}+(1-3 \alpha) z^{2}}{1-z^{2}}>0 .
$$

Since $\left|1-z^{2}\right|^{2} \neq 0$, for all $z$ in $U, 0 \leq \alpha \leq 1$ and

$$
\begin{aligned}
& \left(\left(1-\bar{z}^{2}\right)\left(1+\alpha z^{4}+(1-3 \alpha) z^{2}\right)\right) \\
= & \left(1-\bar{z}^{2}+z^{2}-|z|^{4}+\alpha\left(z^{4}-3 z^{2}+3|z|^{4}-|z|^{4} z^{2}\right)\right),
\end{aligned}
$$

it follows that

$$
\operatorname{Re}(\Psi(z))=\operatorname{Re} \frac{\left(1-\bar{z}^{2}+z^{2}-|z|^{4}+\alpha\left(z^{4}-3 z^{2}+3|z|^{4}-|z|^{4} z^{2}\right)\right)}{\left|1-z^{2}\right|^{2}} .
$$

Note that $\Psi(0)=1$ and for each $\gamma \in \mathbb{R}$, 


$$
\begin{aligned}
& \operatorname{Re}\left(\Psi\left(e^{i \gamma}\right)\right)=\operatorname{Re} \frac{\left(1-\left(e^{-i \gamma}\right)^{2}+\left(e^{i \gamma}\right)^{2}-\left|e^{i \gamma}\right|^{4}+\alpha\left(\left(e^{i \gamma}\right)^{4}-3\left(e^{i \gamma}\right)^{2}+3\left|e^{i \gamma}\right|^{4}-\left|e^{i \gamma}\right|^{4}\left(e^{i \gamma}\right)^{2}\right)\right)}{\left|1-\left(e^{i \gamma}\right)^{2}\right|^{2}} \\
& =\operatorname{Re} \frac{\left(1-\left(e^{-i \gamma}\right)^{2}+\left(e^{i \gamma}\right)^{2}-1+\alpha\left(\left(e^{i \gamma}\right)^{4}-3\left(e^{i \gamma}\right)^{2}+3-\left(e^{i \gamma}\right)^{2}\right)\right)}{\left|1-\left(e^{i \gamma}\right)^{2}\right|^{2}} \\
& =\operatorname{Re} \frac{\left(-4 i \cos (\gamma) \sin (\gamma)+\alpha\left(\left(e^{i \gamma}\right)^{4}-3\left(e^{i \gamma}\right)^{2}+3-\left(e^{i \gamma}\right)^{2}\right)\right)}{\left|1-\left(e^{i \gamma}\right)^{2}\right|^{2}} \\
& =\operatorname{Re} \frac{\left(i(\sin (4 \gamma-4 \sin (2 \gamma))-4 \cos (\gamma) \sin (\gamma))+2 \alpha\left((\cos (2 \gamma)-1)^{2}\right)\right)}{\left|1-\left(e^{i \gamma}\right)^{2}\right|^{2}} \\
& =\frac{2 \alpha}{\left|1-\left(e^{i \gamma}\right)^{2}\right|^{2}}\left((\cos (2 \gamma)-1)^{2}\right) \geq 0, \quad 0 \leq \alpha \leq 1 .
\end{aligned}
$$

Hence, by the minimum principle for harmonic functions, we obtain

$$
\operatorname{Re}(\Psi(z))=\operatorname{Re}\left(\left(1-z^{2}\right) K_{\alpha}^{\prime}(z)\right)>0,
$$

for all $z \in U$, and this yields the result.

In addition, by Lemma 1 , we can deduce that the analytic function $K_{\alpha}=h_{\alpha}+g_{\alpha}$ is univalent and maps onto domain convex in the direction of the imaginary axis. Further, Theorem 3 yields that the harmonic mapping $h_{\alpha}+\bar{g}_{\alpha}$ belongs to the class $S_{H}$ and is convex in the direction of imaginary axis. Figure 8 gives an illustration of images of $U$ under $K_{\alpha}$ for several values of $\alpha$.

Let us begin by presenting our own dilatation $\omega$.

Theorem 7. Let $f_{\alpha_{j}}=h_{\alpha_{j}}+\bar{g}_{\alpha_{j}}$, for $j=1,2$, be two normalized harmonic mapping satisfying $h_{\alpha_{j}}(z)+g_{\alpha_{j}}(z)=$ $z\left(1-\alpha_{j} z^{2}\right) /\left(1-z^{2}\right), 0 \leq \alpha_{j} \leq 1$, and dilatation $\omega_{j}=g_{j}^{\prime} / h_{j}^{\prime}$ with $\left|\omega_{j}\right|<1$ in $U$. Then, the second complex dilatation $\omega$ of the mapping $f=t f_{\alpha_{1}}+(1-t) f_{\alpha_{2}}, 0 \leq t \leq 1$, is defined to be

$$
\omega(z)=\frac{\left(1+z^{2}\right)\left(\omega_{1} \omega_{2}+t \omega_{1}+(1-t) \omega_{2}\right)+z^{2}\left(z^{2}-3\right)\left(\alpha_{1} t \omega_{1}\left(1+\omega_{2}\right)+\alpha_{2}(1-t)\left(1+\omega_{1}\right) \omega_{2}\right)}{\left(1+z^{2}\right)\left(1+t \omega_{2}+(1-t) \omega_{1}\right)+z^{2}\left(z^{2}-3\right)\left(\alpha_{1} t\left(1+\omega_{2}\right)+\alpha_{2}(1-t)\left(1+\omega_{1}\right)\right)} .
$$

Proof. Since

$f=t f_{\alpha_{1}}+(1-t) f_{\alpha_{2}}=t h_{\alpha_{1}}+(1-t) h_{\alpha_{2}}+t \bar{g}_{\alpha_{1}}+(1-t) \bar{g}_{\alpha_{2}}$, it follows that the dilatation $\omega$ of the mapping $f$ is given by

$$
\omega=\frac{t g_{\alpha_{1}}{ }^{\prime}+(1-t) g_{\alpha_{2}}{ }^{\prime}}{t h_{\alpha_{1}}{ }^{\prime}+(1-t) h_{\alpha_{2}}{ }^{\prime}}=\frac{t \omega_{1} h_{\alpha_{1}}{ }^{\prime}+(1-t) \omega_{2} h_{\alpha_{2}}{ }^{\prime}}{t h_{\alpha_{1}}{ }^{\prime}+(1-t) h_{\alpha_{2}}{ }^{\prime}}
$$

Making use of the mapping $h_{\alpha_{j}}(z)+g_{\alpha_{j}}(z)=$ $z\left(1-\alpha_{j} z^{2}\right) /\left(1-z^{2}\right)$ and $\omega_{j}=g_{\alpha_{j}}{ }^{\prime} / h_{\alpha_{j}}{ }^{\prime}$, for $j=1,2$, we obtain

$$
\begin{aligned}
& h_{\alpha_{1}}{ }^{\prime}(z)=\frac{1+z^{2}\left(1+\alpha_{1}\left(z^{2}-3\right)\right)}{\left(1+\omega_{1}\right)\left(1-z^{2}\right)^{2}}, \\
& h_{\alpha_{2}}{ }^{\prime}(z)=\frac{1+z^{2}\left(1+\alpha_{2}\left(z^{2}-3\right)\right)}{\left(1+\omega_{2}\right)\left(1-z^{2}\right)^{2}} .
\end{aligned}
$$

Replacing the above expressions with $h_{\alpha_{1}}{ }^{\prime}$ and $h_{\alpha_{2}}{ }^{\prime}$ in (57), it follows that 


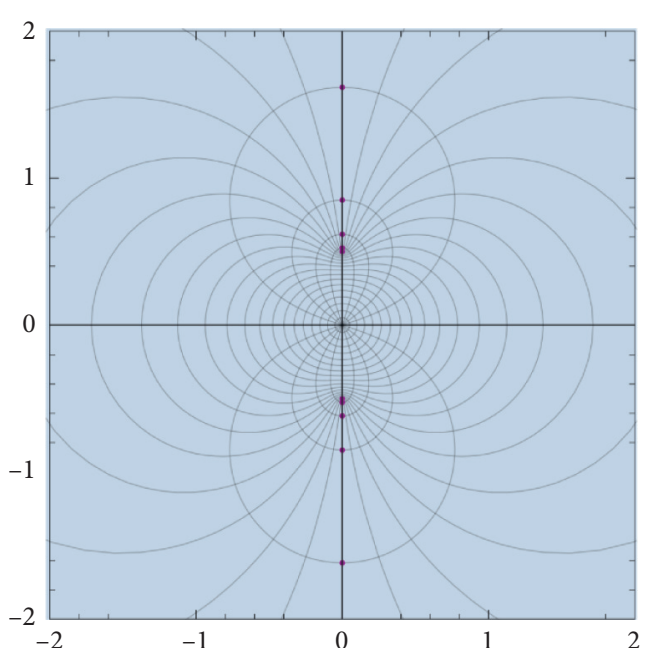

(a)

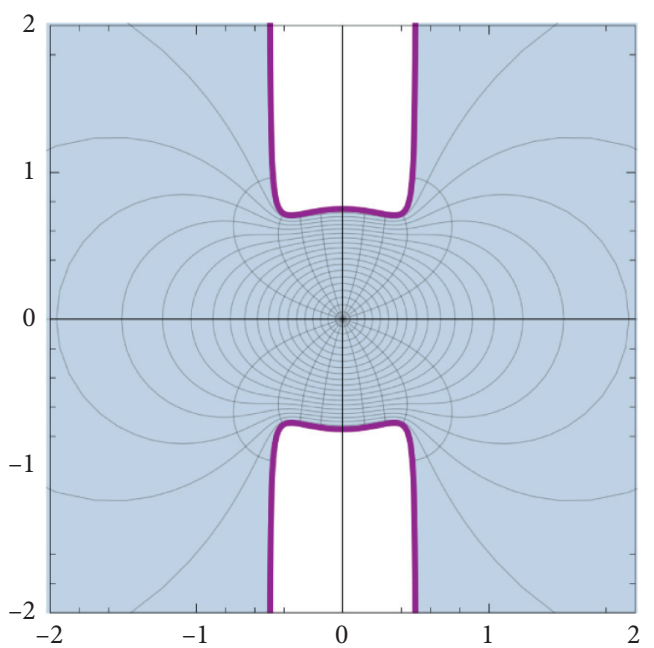

(c)

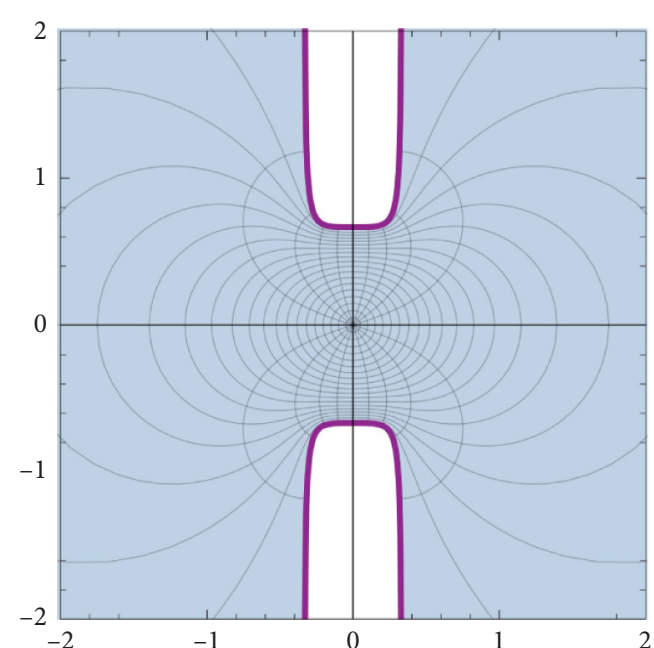

(b)

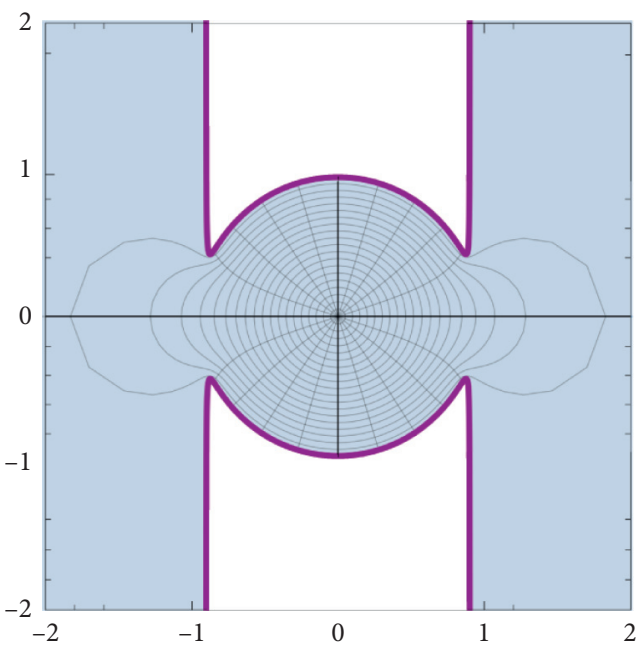

(d)

Figure 8: Images of (a) $K_{0}(U)$, (b) $K_{1 / 3}(U)$, (c) $K_{1 / 2}(U)$, and (d) $K_{0.9}(U)$.

$$
\begin{aligned}
\omega & =\frac{t \omega_{1}\left(1+z^{2}\left(1+\alpha_{1}\left(z^{2}-3\right)\right) /\left(1+\omega_{1}\right)\left(1-z^{2}\right)^{2}\right)+(1-t) \omega_{2}\left(1+z^{2}\left(1+\alpha_{2}\left(z^{2}-3\right)\right) /\left(1+\omega_{2}\right)\left(1-z^{2}\right)^{2}\right)}{t\left(1+z^{2}\left(1+\alpha_{1}\left(z^{2}-3\right)\right) /\left(1+\omega_{1}\right)\left(1-z^{2}\right)^{2}\right)+(1-t)\left(1+z^{2}\left(1+\alpha_{2}\left(z^{2}-3\right)\right) /\left(1+\omega_{2}\right)\left(1-z^{2}\right)^{2}\right)} \\
& =\frac{t \omega_{1}\left(1+z^{2}+\alpha_{1} z^{2}\left(z^{2}-3\right)\right)\left(1+\omega_{2}\right)+(1-t) \omega_{2}\left(1+z^{2}+\alpha_{2} z^{2}\left(z^{2}-3\right)\right)\left(1+\omega_{1}\right)}{t\left(1+z^{2}+\alpha_{1} z^{2}\left(z^{2}-3\right)\right)\left(1+\omega_{2}\right)+(1-t)\left(1+z^{2}+\alpha_{2} z^{2}\left(z^{2}-3\right)\right)\left(1+\omega_{1}\right)},
\end{aligned}
$$

which yields the results in (56) through some computation.

The next result clarifies that the necessary and sufficient condition for the convex combination of $f_{\alpha_{1}}$ and $f_{\alpha_{2}}$ is in the class $S_{H}$ and convex in the direction of the imaginary axis if it is locally univalent and sense-preserving.

Theorem 8. Let $f_{\alpha_{j}}=h_{\alpha_{j}}+\bar{g}_{\alpha_{j}}$, for $j=1,2$, be two normalized harmonic mapping satisfying $h_{\alpha_{j}}(z)+g_{\alpha_{j}}(z)=$ $z\left(1-\alpha_{j} z^{2}\right) /\left(1-z^{2}\right), 0 \leq \alpha_{j} \leq 1$, and dilatation $\omega_{j}=g_{\alpha_{j}}^{\prime} / h_{\alpha_{j}}^{\prime}$ with $\left|\omega_{j}\right|<1$ in $U$. Then, the mapping $f=t f_{\alpha_{1}}+(1-t) f_{\alpha_{2}}$, $0 \leq t \leq 1$ with dilatation $\omega$ as given in (56), is in $S_{H}$ and maps into region convex in the direction of the imaginary axis, provided $f$ is locally univalent and sense-preserving.

Proof. Define

$$
f=h+\bar{g}=\left(t h_{\alpha_{1}}+(1-t) h_{\alpha_{2}}\right)+\overline{t g_{\alpha_{1}}+(1-t) g_{\alpha_{2}}} .
$$

Let 


$$
\begin{aligned}
h+g & =t\left(h_{\alpha_{1}}+g_{\alpha_{1}}\right)+(1-t)\left(h_{\alpha_{2}}+g_{\alpha_{2}}\right) \\
& =t\left(h_{\alpha_{1}}+g_{\alpha_{1}}\right)+(1-t)\left(h_{\alpha_{2}}+g_{\alpha_{2}}\right) \\
& =t K_{\alpha_{1}}+(1-t) K_{\alpha_{2}},
\end{aligned}
$$

where $K_{\alpha_{j}}=h_{\alpha_{j}}+g_{\alpha_{j}}, j=1,2$. Since the mapping $f_{\alpha_{j}}$ satisfies (49), it follows from (7) that

$$
\begin{aligned}
\operatorname{Re}\left\{\left(1-z^{2}\right)\left(h^{\prime}+g^{\prime}\right)\right\}= & t \operatorname{Re}\left\{\left(1-z^{2}\right) K_{\alpha_{1}}{ }^{\prime}\right\} \\
& +(1-t) \operatorname{Re}\left\{\left(1-z^{2}\right) K_{\alpha_{2}}^{\prime}\right\}>0,
\end{aligned}
$$

on $U$ for all $0 \leq t \leq 1$. Hence, according to Lemma 1 , one can deduce that the mapping $h+g$ is univalently convex in the direction of the imaginary axis. Also, If $f=h+\bar{g}$ is sense- preserving locally univalent mapping in $U$, then in view of Theorem 3, $f \in S_{H}$ is univalent analytic mapping of $U$ onto a region convex in the direction of imaginary axis.

Theorem 9. For $j=1,2$, define $f_{\alpha_{j}}=h_{\alpha_{j}}+\bar{g}_{\alpha_{j}}$ to be normalized sense-preserving harmonic mapping such that $h_{\alpha_{j}}(z)+g_{\alpha_{j}}(z)=z\left(1-\alpha_{j} z^{2}\right) /\left(1-z^{2}\right), 0 \leq \alpha_{j} \leq 1$ with $\alpha_{j}=$ $\alpha$. Let $\omega_{j}$ be the dilatation of $f_{\alpha_{j}}\left(\left|\omega_{j}\right|<1\right.$ in $\left.U\right)$. If $\omega_{1} \neq \omega_{2}$, then the mapping $f=t f_{\alpha_{1}}+(1-t) f_{\alpha_{2}}, 0 \leq t \leq 1$, is in $S_{H}$ and is convex in the direction of the imaginary axis.

Proof. From Theorem 8, it will be sufficient if we show that the dilatation of $f$ given in (56) satisfies the condition $|\omega|<1$ in $U$. Suppose that $\alpha_{1}=\alpha_{2}=\alpha$ in (56); then,

$$
\begin{aligned}
\omega & =\frac{\left(1+z^{2}\right)\left[\omega_{1} \omega_{2}+t \omega_{1}+(1-t) \omega_{2}\right]+\alpha z^{2}\left(z^{2}-3\right)\left[\omega_{1} \omega_{2}+t \omega_{1}+(1-t) \omega_{2}\right]}{\left(1+z^{2}\right)\left[1+t \omega_{2}+(1-t) \omega_{1}\right]+\alpha z^{2}\left(z^{2}-3\right)\left[1+t \omega_{2}+(1-t) \omega_{1}\right]} \\
& =\frac{\left(\omega_{1} \omega_{2}+t \omega_{1}+(1-t) \omega_{2}\right)\left(1+\alpha z^{4}+(1-3 \alpha) z^{2}\right)}{\left(1+t \omega_{2}+(1-t) \omega_{1}\right)\left(1+z^{2}+\alpha z^{2}\left(z^{2}-3\right)\right)} \\
& =\frac{\omega_{1} \omega_{2}+t \omega_{1}+(1-t) \omega_{2}}{1+t \omega_{2}+(1-t) \omega_{1}} .
\end{aligned}
$$

From the proof of Theorem 3 in [21], it is clear that $|\omega|<1$. Hence, we deduce that the mapping $f$ is locally univalent and sense-preserving.

Next, we will consider one of the harmonic mappings involved in the linear combination induced by shearing analytic mapping $z(1-\beta z) /\left(1-z^{2}\right)$ where $\beta \in[-1,1]$.

Theorem 10. Define $f_{\beta}=h_{\beta}+\bar{g}_{\beta}$, satisfying $h_{\beta}(z)+g_{\beta}(z)$ $=z(1-\beta z) /\left(1-z^{2}\right),-1 \leq \beta \leq 1$, with dilatation $\omega_{1}=g_{\beta}^{\prime} / h_{\beta}^{\prime}$ lies in $U$ and let $f_{\alpha}=h_{\alpha}+\bar{g}_{\alpha}$, satisfying $h_{\alpha}(z)+g_{\alpha}(z)=$ $z\left(1-\alpha z^{2}\right) /\left(1-z^{2}\right), 0 \leq \alpha \leq 1$, with dilatation $\omega_{2}=g_{\alpha}^{\prime} / h_{\alpha}^{\prime}$ lies in $U$, be two normalized harmonic mapping. Then, the mapping $f_{\beta, \alpha}=t f_{\beta}+(1-t) f_{\alpha}, 0 \leq t \leq 1$ is univalent and convex in the direction of the imaginary axis if $f_{\beta, \alpha}$ is locally univalent and sense-preserving.

Proof. Since $h_{\alpha}(z)+g_{\alpha}(z)=z\left(1-\alpha z^{2}\right) /\left(1-z^{2}\right)$ and $h_{\beta}(z)$ $+h_{\beta}(z)=z(1-\beta z) /\left(1-z^{2}\right)$, it follows that

$$
\begin{aligned}
h+g & =t h_{\alpha}+(1-t) h_{\beta}+t g_{\alpha}+(1-t) g_{\beta} \\
& =t\left(h_{\alpha}+g_{\alpha}\right)+(1-t)\left(h_{\beta}+g_{\beta}\right),
\end{aligned}
$$

which implies that

$$
h^{\prime}+g^{\prime}=t\left(h_{\alpha}^{\prime}+g_{\alpha}^{\prime}\right)+(1-t)\left(h_{\beta}^{\prime}+g_{\beta}^{\prime}\right) .
$$

Hence,

$$
\begin{aligned}
\operatorname{Re}\left\{\left(1-z^{2}\right)\left(h^{\prime}+g^{\prime}\right)\right\}= & t \operatorname{Re}\left\{\left(1-z^{2}\right)\left(h_{\alpha}^{\prime}+g_{\alpha}^{\prime}\right)\right\} \\
& +(1-t) \operatorname{Re}\left\{\left(1-z^{2}\right)\left(h_{\beta}^{\prime}+g_{\beta}^{\prime}\right)\right\}
\end{aligned}
$$

from (55). Since

$$
\operatorname{Re}\left\{\left(1-z^{2}\right)\left(h_{\beta}^{\prime}+g_{\beta}^{\prime}\right)\right\}=\frac{\left(1-\left|z^{2}\right|\right)\left(1+|z|^{2}-2 \beta \operatorname{Re}(z)\right)}{\left|1-z^{2}\right|^{2}}>0,
$$

for all $0 \leq t \leq 1$, we can easily see that

$$
\operatorname{Re}\left\{\left(1-z^{2}\right)\left(h^{\prime}+g^{\prime}\right)\right\}>0 .
$$

In view of Theorem 3, we deduce that the mapping $f=$ $h+\bar{g}$ is univalent and maps onto domain convex in the direction of the imaginary axis provided $f$ is locally univalent and sense-preserving on $U$.

It is known that for $0 \leq \theta<\pi$, the mapping $f_{\theta}=h_{\theta}+\bar{g}_{\theta}$ is called vertical strip mapping if it satisfies

$$
h_{\theta}(z)+g_{\theta}(z)=\frac{1}{2 i \sin \theta} \log \left(\frac{1+e^{i \theta} z}{1-e^{i \theta} z}\right) .
$$

Thus, Theorem 10 can be expressed in terms of vertical strip mappings. 
Corollary 2. Let $f_{\theta}=h_{\theta}+\bar{g}_{\theta}$, where $h_{\theta}(z)+g_{\theta}(z)$ $=1 / 2 i \sin \theta \log \left(1+e^{i \theta} z / 1-e^{i \theta} z\right)$ with dilatation $g_{\theta}^{\prime} / h_{\theta}^{\prime}$ lies in $U$ and $f_{\alpha}=h_{\alpha}+\bar{g}_{\alpha}$, where $h_{\alpha}(z)+g_{\alpha}(z)=z\left(1-\alpha z^{2}\right) /$ $\left(1-z^{2}\right), 0 \leq \alpha \leq 1$ with dilatation $g_{\alpha}^{\prime} / h_{\alpha}^{\prime}$ lies in $U$, to be two normalized harmonic mapping. Then, the mapping $f_{\theta, \alpha}=t f_{\theta}+(1-t) f_{\alpha}, 0 \leq t \leq 1$, is univalent and convex in the direction of the imaginary axis if $f_{\theta, \alpha}$ is locally univalent and sense-preserving.

\section{Data Availability}

No data were used to support this study.

\section{Conflicts of Interest}

The authors declare that they have no conflicts of interest.

\section{Acknowledgments}

The first and second authors were supported by the Fundamental Research Grant Scheme (Ministry of Education Malaysia (MOE), Acct No. 203.PMATHS.6711939).

\section{References}

[1] H. Lewy, "On the non-vanishing of the Jacobian in certain one-to-one mappings," Bulletin of the American Mathematical Society, vol. 42, no. 10, pp. 689-693, 1936.

[2] D. Peter, Harmonic Mappings in the Plane, Cambridge University Press, Cambridge, UK, 2004.

[3] M. Dorff, "Convolutions of planar harmonic convex mappings," Complex Variables, vol. 45, pp. 263-271, 2001.

[4] S. Ruscheweyh and T. Sheil-Small, "Hadamard products of schlicht functions and the Polya-Schoenberg conjecture," Commentarii Mathematici Helvetici, vol. 48, pp. 119-135, 1973.

[5] S. Beig and V. Ravichandran, "Convolution and convex combination of harmonic mappings," Bulletin of the Iranian Mathematical Society, vol. 45, no. 5, pp. 1467-1486, 2019.

[6] R. Kumar, S. Gupta, S. Singh, and M. Dorff, "An application of Cohn's rule to convolutions of univalent harmonic mappings," Rocky Mountain Journal of Mathematics, vol. 46, no. 2, pp. 559-570, 2016.

[7] Z.-H. Liu and Y.-C. Li, "The properties of a new subclass of harmonic univalent mappings," Abstract and Applied Analysis, vol. 2013, Article ID 794108, 7 pages, 2013.

[8] Z. Liu and S. Ponnusamy, "Univalency of convolutions of univalent harmonic right half-plane mappings," Computational Methods and Function Theory, vol. 17, no. 2, pp. 289302, 2017.

[9] M. Dorff, M. Nowak, and M. Woloszkiewicz, "Convolutions of harmonic convex mappings," Complex Variables and Elliptic Equations, vol. 57, pp. 489-503, 2012.

[10] M. Goodloe, "Hadamard products of harmonic mappings," Complex Variable Theory and Applications, vol. 47, p. 8192, 2002.

[11] L. Li and S. Ponnusamy, "Solution to an open problem on convolutions of harmonic mappings," Complex Variables and Elliptic Equations, vol. 58, pp. 1647-1653, 2013.

[12] R. Kumar, M. Dorff, S. Gupta, and S. Singh, "Convolution properties of some harmonic mappings in the right halfplane," The Bulletin of the Malaysian Mathematical Society Series 2, vol. 39, no. 1, pp. 439-455, 2016.
[13] J. Clunie and T. Sheil-Small, "Harmonic univalent functions," Annales Academiae Scientiarum Fennicae Series A I Mathematica, vol. 9, pp. 3-25, 1984.

[14] M. F. Ali, V. Allu, and N. Ghosh, "A convolution property of univalent harmonic right half-plane mappings," Monatshefte für Mathematik, vol. 193, no. 4, pp. 729-736, 2020.

[15] W. Hengartner and G. Schober, "On Schlicht mappings to domains convex in one direction," Commentarii Mathematici Helvetici, vol. 45, no. 1, pp. 303-314, 1970.

[16] C. Pommerenke, "On starlike and close-to-convex functions," Proceedings of the London Mathematical Society, vol. s3-13, no. 1, pp. 290-304, 1963.

[17] M. J. Dorff and J. S. Rolf, "Anamorphosis, mapping problems, and harmonic univalent functions," Explorations in Complex Analysis, pp. 197-269, Mathematical Association of America, Washington, DC, USA, 2012.

[18] T. H. MacGregor, "The univalence of a linear combination of convex mappings," Journal of the London Mathematical Society, vol. s1-44, no. 1, pp. 210-212, 1969.

[19] L. Shi, Z.-G. Wang, A. Rasila, and Y. Sun, "Convex combinations of harmonic shears of slit mappings," Bulletin of the Iranian Mathematical Society, vol. 43, no. 5, pp. 1495-1510, 2017.

[20] R. Kumar, S. Gupta, and S. Singh, "Linear combinations of univalent harmonic mappings convex in the direction of the imaginary axis," Bulletin of the Malaysian Mathematical Sciences Society, vol. 39, no. 2, pp. 751-763, 2016.

[21] Z.-G. Wang, Z.-H. Liu, and Y.-C. Li, "On the linear combinations of harmonic univalent mappings," Journal of Mathematical Analysis and Applications, vol. 400, no. 2, pp. 452-459, 2013.

[22] Á. Ferrada-Salas, R. Hernández, and M. J. Martín, “On convex combinations of convex harmonic mappings," Bulletin of the Australian Mathematical Society, vol. 96, no. 2, pp. 256-262, 2017.

[23] S. Beig, Y. J. Sim, and N. E. Cho, "On convex combinations of harmonic mappings," Journal of Inequalities and Applications, vol. 2020, p. 14, 2020.

[24] Q. I. Rahman and G. Schmeisser, Analytic Theory of Polynomials, Vol. 26, Oxford University Press, Oxford, UK, 2002. 